\title{
Quantum marking and quantum erasure for neutral kaons
}

\author{
A. Bramon ${ }^{1}$, G. Garbarino ${ }^{2}$ and B. Hiesmayr ${ }^{1}$ \\ ${ }^{1}$ Grup de Física Teòrica, Universitat Autònoma de Barcelona, E-08193 Bellaterra, Spain \\ ${ }^{2}$ Departament d'Estructura i Constituents de la Matèria, Universitat de Barcelona, E-08028 Barcelona, Spain
}

(November 8, 2018)

\begin{abstract}
Entangled $K^{0} \bar{K}^{0}$ pairs are shown to be suitable to discuss extensions and tests of Bohr's complementarity principle through the quantum marking and quantum erasure techniques suggested by M. O. Scully and K. Drühl [Phys. Rev. A 25, 2208 (1982)]. Strangeness oscillations play the role of the traditional interference pattern linked to wave-like behaviour, whereas the distinct propagation in free space of the $K_{S}$ and $K_{L}$ components mimics the two possible interferometric paths taken by particle-like objects.
\end{abstract}

PACS numbers: 03.65.-w, 14.40.Aq

Some twenty years ago Scully and Drühl [1] discussed an interesting gedanken experiment concerning the possibility to erase information contained in quantum states and the effects that this erasure can have on measurement outcomes. Further refinements of the original proposal have appeared in recent years (see, for instance, Refs. [2,3]) and its connection with a central feature of quantum mechanics - Bohr's complementarity principle - has been widely debated. The quantum mechanical predictions, including quantitative descriptions of complementarity [4], have been confirmed by a variety of recent interferometric experiments with atoms [5] or photon pairs [6-14].

In this type of analyses one considers variations on the basic double-slit experiment and exploits the well known complementarity between the observation of interference fringes (wave-like behaviour) and the acquisition of "which way" information (particle-like behaviour). Interference patterns are observed if and only if in the twoway experiment it is impossible to know, even in princi$p l e$, which way the particle took. Interference disappears if there is a way to know - e.g., through a quantum marking procedure - which way the particle took; whether or not the outcome of the corresponding "which way" observation is actually read out, it does not matter: interference is in any way lost.

In most of the experiments performed up to date [6-14] one uses two-photon entangled states from spontaneous parametric down-conversion (SPDC). If the path of one photon is marked, information on the path taken by its entangled partner is in principle available and no interference fringes can be observed. But, if that "which way" mark (usually, a specific polarization) is erased by means of a suitable measurement — quantum erasure - , observation of interference fringes becomes possible in joint detection events.

The purpose of this Letter is to extend these considerations to entangled pairs of neutral kaons. A neutral kaon beam presents the well known phenomenon of $K^{0}-\bar{K}^{0}$ oscillations (in time), which will play the role of the pho- ton interference fringes (in space). Similarly, the shortand long-lived kaon states, $K_{S}$ and $K_{L}$, showing a distinct propagation in free-space, are the analogs of the two separated photon trajectories in interferometric devices. New forms of quantum markers and erasers are thus offered and Bohr's complementary principle extends its applicability. The CPLEAR experiment [15], with $K^{0} \overline{K^{0}}$ pairs created in $p \bar{p}$ annihilations at rest, can be interpreted as a preliminary quantum eraser experiment. The presently operating DA $\Phi N E \phi$-factory [16] offers the possibility to perform a complete test of complementarity by investigating both quantum marking and erasure along the lines we discuss in this note.

Two alternative bases, each one associated to a possible measurement on a neutral kaon, have to be considered [17]. The strangeness basis, $\left\{K^{0}, \bar{K}^{0}\right\}$ with $\left\langle K^{0} \mid \bar{K}^{0}\right\rangle=0$, is the appropriate one to discuss strong production and reactions of kaons, as well as kaon strangeness measurements. Indeed, if a dense piece of ordinary (nucleonic) matter is inserted along the neutral kaon trajectory, the incoming state is projected either into $K^{0}$ (by strangeness conserving strong reactions such as $K^{0} p \rightarrow$ $\left.K^{+} n\right)$ or into $\bar{K}^{0}\left(\bar{K}^{0} p \rightarrow \Lambda \pi^{+}, \bar{K}^{0} n \rightarrow \Lambda \pi^{0}, \bar{K}^{0} n \rightarrow\right.$ $\left.K^{-} p\right)$. Nucleonic matter plays here the same role as a two-channel analyzer for polarized photon beams.

The second basis consists of the $K_{S}$ and $K_{L}$ states having well defined masses $m_{S(L)}$ and decay widths $\Gamma_{S(L)}$; it is the appropriate one to discuss neutral kaon propagation in free space, with:

$$
\left|K_{S(L)}(\tau)\right\rangle=e^{-i \lambda_{S(L)} \tau}\left|K_{S(L)}\right\rangle,
$$

and $\lambda_{S(L)}=m_{S(L)}-i \Gamma_{S(L)} / 2$. The $K_{S}$ and $K_{L}$ eigenstates preserve their own identity with (proper) time $\tau$, but, since $\Gamma_{S} \simeq 579 \Gamma_{L}$, the short-lived component of a given neutral kaon extincts much faster than its longlived one. Knowing if this kaon has propagated in freespace either as $K_{S}$ or $K_{L}$ is thus possible by detecting at which time it decays. If kaons decaying before $\simeq 4.8 \tau_{S}$ after production are identified as $K_{S}$ 's and those surviving after this time as $K_{L}$ 's, misidentifications amount to 
only to a few parts in $10^{-3}[17,18]$. Such a discrimination between the $K_{S}$ and $K_{L}$ propagation modes, linked to the obtention of "which width" information, is thus in analogy with usual "which way" discussions in photon experiments.

The relationship between these two kaon bases is well known. Although one strictly has $\left\langle K_{S} \mid K_{L}\right\rangle=$ $2(\operatorname{Re} \epsilon) /\left(1+|\epsilon|^{2}\right) \simeq 3.2 \times 10^{-3}$, one can neglect this small CP violating effect [19] because it is of the same order as the previously mentioned $K_{S}$ vs $K_{L}$ misidentifications and escapes from our control. With usual conventions [19], one can then approximate:

$$
\begin{aligned}
\left|K^{0}\right\rangle & =\left[\left|K_{S}\right\rangle+\left|K_{L}\right\rangle\right] / \sqrt{2}, \\
\left|\bar{K}^{0}\right\rangle & =\left[\left|K_{S}\right\rangle-\left|K_{L}\right\rangle\right] / \sqrt{2},
\end{aligned}
$$

and the two bases are associated with two (in a sense, maximally) incompatible observables. Indeed, the proposed strangeness measurements and lifetime observations completely exclude each other: the former require the insertion of nucleonic matter, the latter propagation in free-space. Bohr's complementarity principle is thus at work: if strangeness (lifetime) is known, both outcomes for lifetime (strangeness) are equally probable, as follows immediately from Eqs. (2).

Suppose that at time $\tau=0$ a $K^{0}$ is produced via the strong reaction $\pi^{-} p \rightarrow K^{0} \Lambda$. From Eqs. (1) and (2), at proper time $\tau$ one has the following transition probabilities $\left(\Delta m \equiv m_{L}-m_{S}\right)$ :

$$
\begin{aligned}
\left|\left\langle K^{0} \mid K^{0}(\tau)\right\rangle\right|^{2}= & \left\{e^{-\Gamma_{S} \tau}+e^{-\Gamma_{L} \tau}\right\} \\
& \times\left\{1+\mathcal{V}_{0}(\tau) \cos (\Delta m \tau)\right\} / 4, \\
\left|\left\langle\bar{K}^{0} \mid K^{0}(\tau)\right\rangle\right|^{2}= & \left\{e^{-\Gamma_{S} \tau}+e^{-\Gamma_{L} \tau}\right\} \\
& \times\left\{1-\mathcal{V}_{0}(\tau) \cos (\Delta m \tau)\right\} / 4, \\
\left|\left\langle K_{L} \mid K^{0}(\tau)\right\rangle\right|^{2}= & \left|\left\langle K_{L} \mid K_{L}(\tau)\right\rangle\right|^{2} / 2=e^{-\Gamma_{L} \tau} / 2, \\
\left|\left\langle K_{S} \mid K^{0}(\tau)\right\rangle\right|^{2}= & \left|\left\langle K_{S} \mid K_{S}(\tau)\right\rangle\right|^{2} / 2=e^{-\Gamma_{S} \tau} / 2 .
\end{aligned}
$$

Eqs. (3) and (4) show the well known strangeness oscillation phenomena with a time dependent "fringe visibility" $\mathcal{V}_{0}(\tau)=1 / \cosh (\Delta \Gamma \tau / 2)$, where $\Delta \Gamma \equiv \Gamma_{L}-\Gamma_{S}$. On the contrary, Eqs. (5) and (6) show that no $K_{S}-K_{L}$ oscillations are expected.

These observations admit the following interpretation. As soon as a $K^{0}$ is produced, it starts propagating in free space in the coherent superposition of $K_{S}$ and $K_{L}$ given by Eqs. (2) and mimics the two-way propagation of any system beyond a symmetrical double-slit. In the familiar double-slit case, the system follows the two paths without "jumping" from one to the other, in the same way as $K_{S}-K_{L}$ oscillations are "forbidden". In the kaon case, however, there are not two separated trajectories but a single path comprising automatically (i.e., with no need of any double-slit like apparatus) the two differently propagating components $K_{S}$ and $K_{L}$. At $\tau=0$ there is no information on which component actually propagates ["predictability" of the path [4], $\mathcal{P}(\tau=0)=0$ ] and the visibility of strangeness oscillations is maximal, $\mathcal{V}_{0}(\tau=0)=1$. However, since the $K_{S}$ and $K_{L}$ components are intrinsically "marked" by their different lifetimes, "which width" information is obtained for initial $K^{0}$ s surviving up to $\tau$ and the corresponding oscillation visibility is now reduced. One easily finds $\mathcal{P}(\tau)=|\tanh (\Delta \Gamma \tau / 2)|$, thus fulfilling the quantitative duality requirement $\mathcal{V}_{0}^{2}(\tau)+\mathcal{P}^{2}(\tau)=1[4]$ and supporting our present interpretation.

Working with entangled kaon pairs, in the same way as one uses entangled pairs of photons in analogous optical experiments, one can perform quantum marking and erasure. To this aim, consider the decay of the $\phi(1020)-$ meson resonance [16] (or, alternatively, $S$-wave $p \bar{p}$ annihilation [15]) into $K^{0} \bar{K}^{0}$ pairs. Just after the decay $(\tau=0)$ one has the following maximally entangled state:

$$
\begin{aligned}
|\phi(0)\rangle & =\frac{1}{\sqrt{2}}\left[\left|K^{0}\right\rangle_{l}\left|\bar{K}^{0}\right\rangle_{r}-\left|\bar{K}^{0}\right\rangle_{l}\left|K^{0}\right\rangle_{r}\right] \\
& =\frac{1}{\sqrt{2}}\left[\left|K_{L}\right\rangle_{l}\left|K_{S}\right\rangle_{r}-\left|K_{S}\right\rangle_{l}\left|K_{L}\right\rangle_{r}\right],
\end{aligned}
$$

where $l$ and $r$ denote the "left" and "right" kaon directions of motion. In the lifetime basis, the state evolution up to time $\tau_{l}\left(\tau_{r}\right)$ along the left (right) beam is given by:

$$
\begin{aligned}
&\left|\phi\left(\tau_{l}, \tau_{r}\right)\right\rangle=\frac{1}{\sqrt{2}}\left\{e^{-i\left(\lambda_{L} \tau_{l}+\lambda_{S} \tau_{r}\right)}\left|K_{L}\right\rangle_{l}\left|K_{S}\right\rangle_{r}\right. \\
&\left.-e^{-i\left(\lambda_{S} \tau_{l}+\lambda_{L} \tau_{r}\right)}\left|K_{S}\right\rangle_{l}\left|K_{L}\right\rangle_{r}\right\} .
\end{aligned}
$$

It will be convenient to consider only kaon pairs with both left and right members surviving up to $\tau_{l}$ and $\tau_{r}$. These are described by the following normalized state:

$$
\begin{aligned}
|\phi(\Delta \tau)\rangle=\frac{1}{\sqrt{1+e^{\Delta \Gamma \Delta \tau}}}\left\{\left|K_{L}\right\rangle_{l}\left|K_{S}\right\rangle_{r}\right. \\
\left.-e^{i \Delta m \Delta \tau} e^{\frac{1}{2} \Delta \Gamma \Delta \tau}\left|K_{S}\right\rangle_{l}\left|K_{L}\right\rangle_{r}\right\},
\end{aligned}
$$

where $\Delta \tau \equiv \tau_{l}-\tau_{r}$. Eq. (7) can be rewritten as

$$
\begin{gathered}
|\phi(\Delta \tau)\rangle=\frac{1}{\sqrt{2\left(1+e^{\Delta \Gamma \Delta \tau}\right)}}\left\{\left|K^{0}\right\rangle_{l}\left|K_{S}\right\rangle_{r}-\left|\bar{K}^{0}\right\rangle_{l}\left|K_{S}\right\rangle_{r}\right. \\
\left.-e^{i \Delta m \Delta \tau} e^{\frac{1}{2} \Delta \Gamma \Delta \tau}\left[\left|K^{0}\right\rangle_{l}\left|K_{L}\right\rangle_{r}+\left|\bar{K}^{0}\right\rangle_{l}\left|K_{L}\right\rangle_{r}\right]\right\}
\end{gathered}
$$

or, in the strangeness basis, as

$$
\begin{aligned}
& |\phi(\Delta \tau)\rangle=\frac{1}{2 \sqrt{1+e^{\Delta \Gamma \Delta \tau}}} \\
& \times\left\{\left(1-e^{i \Delta m \Delta \tau} e^{\frac{1}{2} \Delta \Gamma \Delta \tau}\right)\left[\left|K^{0}\right\rangle_{l}\left|K^{0}\right\rangle_{r}-\left|\bar{K}^{0}\right\rangle_{l}\left|\bar{K}^{0}\right\rangle_{r}\right]\right. \\
& \left.+\left(1+e^{i \Delta m \Delta \tau} e^{\frac{1}{2} \Delta \Gamma \Delta \tau}\right)\left[\left|K^{0}\right\rangle_{l}\left|\bar{K}^{0}\right\rangle_{r}-\left|\bar{K}^{0}\right\rangle_{l}\left|K^{0}\right\rangle_{r}\right]\right\} .
\end{aligned}
$$

Eqs. (7)-(9) immediately supply the various joint probabilities $P\left(K_{l}, K_{r}\right)$ for detecting a $K_{l}\left(K_{r}\right)$ on the left (right) at time $\tau_{l}\left(\tau_{r}\right)$ with $K_{l, r}=K^{0}, \bar{K}^{0}, K_{S}$ or $K_{L}$. 
By normalizing to surviving kaon pairs, one does not need to consider decay-product states. Thanks to this, the entangled states (7)-(9) only depend on the time difference $\Delta \tau$ and we can work with bipartite two-level quantum systems as in the optical case. For details concerning an accurate description of the kaon pair evolution in $\tau_{l}, \tau_{r}$ see Refs. [20,21].

Quantum marking and erasure for these entangled neutral kaon pairs can be described as follows. One always measures the strangeness of the left moving kaon; by making this measurement at different values of $\tau_{l}$ one can look for strangeness oscillations of this kaon - the signal or object kaon. Conversely, the measurement on the right moving - idler or meter - kaon is always performed at a fixed time $\tau_{r}^{0}$; but, by inserting or not a strangeness detector in two alternative set-ups, either strangeness or lifetime will be measured at $\tau_{r}^{0}$. In this way, various $\tau_{l^{-}}$ dependent probabilities for object-meter joint detections are recorded.

Without the strangeness detector on the right beam, one can observe the decay of the freely propagating meter kaon, which will be identified either as $K_{S}$ or $K_{L}$ [22]. The acquisition of this "which width" information implies the corresponding one for the object kaon and therefore strangeness oscillations (in $\Delta \tau \equiv \tau_{l}-\tau_{r}^{0}$ ) should not be visible for any of the four possible joint detection probabilities $P\left(K_{l}, K_{r}\right)$ with $K_{l}=K^{0}$ or $\bar{K}^{0}$ and $K_{r}=K_{S}$ or $K_{L}$. This is immediately seen from Eq. (8).

However, the possibility to obtain "which width" information can be prevented by quantum erasure, i.e., by measuring strangeness on the meter kaon at time $\tau_{r}^{0}$. The four joint probabilities $P\left(K_{l}, K_{r}\right)$ with $K_{l, r}=K^{0}$ or $\bar{K}^{0}$ will then show the $\tau_{l}$-dependent strangeness oscillations and anti-oscillations immediately deducible from Eq. (9). One has then revived the same $K_{S}-K_{L}$ interference effects typical of the single kaon case [Eqs. (3), (4)], with visibility $\mathcal{V}\left(\tau_{l}\right)=1 / \cosh \left[\Delta \Gamma\left(\tau_{l}-\tau_{r}^{0}\right) / 2\right]$. The erasure is maximal $(\mathcal{V}=1)$ for $\tau_{l}=\tau_{r}^{0}$ (no "which width" information available), but the strangeness oscillations disappear totally $(\mathcal{V} \rightarrow 0)$ for $\tau_{l}-\tau_{r}^{0} \rightarrow \infty$ (full "which width" information available). The time-dependent "distinguishability" of the path [4], quantifying "which width" information, is given by $\mathcal{D}\left(\tau_{l}\right)=\left|\tanh \left[\Delta \Gamma\left(\tau_{l}-\tau_{r}^{0}\right) / 2\right]\right|$ and satisfies the duality relation $\mathcal{V}^{2}\left(\tau_{l}\right)+\mathcal{D}^{2}\left(\tau_{l}\right)=1[4]$.

In a series of optical experiments, entangled pairs of photons have been produced in states similar to that in Eq. (7). In one of the experiments of Ref. [9], two interfering two-photon amplitudes are prepared by forcing a pump beam to cross twice the same non-linear crystal. Idler and signal photons from the first down-conversion are "marked" by rotating their polarization by $90^{\circ}$ and then superposed to the idler and signal photons emerging later from the second passage of the pump beam through the crystal. If type-II SPDC were used, one would obtain the entangled state:

$$
|\Psi\rangle=\left[|V\rangle_{i}|H\rangle_{s}-e^{i \Delta \phi}|H\rangle_{i}|V\rangle_{s}\right] / \sqrt{2}
$$

where the term $|V\rangle_{i}|H\rangle_{s}\left(|H\rangle_{i}|V\rangle_{s}\right)$ refers to photon pairs produced in the second (first) passage and the relative phase $\Delta \phi$ is under control by the experimenter. Thanks to entanglement, the distinct vertical or horizontal idler polarizations supply full "which path" information for the signal photons and no interference can be observed in signal-idler joint detections. To erase this information, the idler (signal) photons have to be detected after crossing polarization analyzers placed at $45^{\circ}$ or $-45^{\circ}\left(45^{\circ}\right)$, i.e., the idler and signal polarizations must be jointly measured in a basis consisting of the two symmetric and antisymmetric superpositions $|H\rangle \pm|V\rangle$ as in Eqs. (2) [23]. Similarly, the photonic experiments of Refs. [6,7] use two SPDC processes and could be discussed along the same lines, but their interpretation as quantum erasers is less clear $[3,8,24]$.

The similarity between the ("which way" marked) twophoton state (10) and the ("which width" marked) twokaon state (7) is obvious. The latter, however, is automatically prepared in $\phi$ decays or $S$-wave $p \bar{p}$ annihilations. These processes can be simply viewed as coherently producing a superposition of the $\left|K_{L}\right\rangle_{l}\left|K_{S}\right\rangle_{r}$ and $\left|K_{S}\right\rangle_{l}\left|K_{L}\right\rangle_{r}$ two-kaon amplitudes. The kaon mass difference $\Delta m$ introduces automatically a time dependent relative phase between the two amplitudes, as it is evident from Eq. (7). The marking and erasure operations can be performed on entangled kaon pairs as in the optical case previously discussed: photon detection after a polarization analyzer at $\theta=45^{\circ}\left(-45^{\circ}\right)$ corresponds to $K^{0}\left(\bar{K}^{0}\right)$ detection, while $\theta=0^{\circ}\left(90^{\circ}\right)$ corresponds to $K_{S}\left(K_{L}\right)$ observation. The additional exponential decay factor, $e^{\frac{1}{2} \Delta \Gamma\left(\tau_{l}-\tau_{r}^{0}\right)}$, which is obviously absent for stable photons, reduces the fringe visibility in kaon experiments.

Another series of photon experiments [10-12] followed a different strategy. Instead of using two SPDC processes, a pump beam is sent to a single non-linear crystal. Each member of the emerging down-converted photon pair is then directed towards a $50 \%-50 \%$ beam-splitter $B S$ in the set-ups of Refs. [10,11]. In Ref. [10], a halfwave plate is inserted along one of the two paths before crossing the $B S$ in order to introduce path distinguishability. In Ref. [11], the two beams emerging from that first $B S$ are sent towards two well separated beamsplitters, thus producing two amplitudes marked by the position of the beam-splitter they come from. In the experiment of Ref. [12], one of the two down-converted photons is directed towards a double-slit: two appropriately placed quarter-wave plates mark each path beyond the double-slit with left or right circular polarizations. In all these experiments [10-12], a two-photon entangled state similar to that in Eq. (10) is obtained after properly postselecting the state directly produced by the interferometers. If these (position or polarization) marks are not erased, interference effects are never seen; by erasing 
with appropriate measurements, which project on symmetric and antisymmetric states as in Eqs. (2), interferences reappear.

Since the $\phi$ resonance essentially consists of a strangeantistrange $s \bar{s}$ quark pair, the decay $\phi \rightarrow K^{0} \bar{K}^{0}$ proceeds by pumping a $d \bar{d}$ pair from the vacuum, which then recombine into a $K^{0}(d \bar{s})-\bar{K}^{0}(s \bar{d})$ meson pair. This decay process, like SPDC from a single non-linear crystal, produces good quality two-particle entangled states. Indeed, each $K^{0} \bar{K}^{0}$ pair produced in a $\phi$ decay automatically propagates in free space as the coherent superposition of $\left|K_{L}\right\rangle_{l}\left|K_{S}\right\rangle_{r}$ and $\left|K_{S}\right\rangle_{l}\left|K_{L}\right\rangle_{r}$ previously discussed. The optical elements (beam-splitters or double slits) needed in the experiments of Refs. [10-12] to produce potentially interfering two-photon amplitudes are automatically provided by Nature for neutral kaons [see Eq. (9)], with no need for postselection.

To summarize, we have explored Bohr's principle in a new direction: the complementarity between strangeness and lifetime measurements on neutral kaons (this closes the issue, since no other projective measurement can be performed). We would like to emphasize that the neutral kaon system reveals to be suitable for an optimal demonstration [2,3] of quantum erasure: (1) since "which width" information is carried by a system (the meter kaon) distinct and spatially separated from the interfering system (the object kaon), the marking and erasure operations can be performed in the "delayed choice" mode $\left(\tau_{l}<\tau_{r}^{0}\right) ;(2)$ single-particle states (as opposed to coherent photon states) are detected on each side; (3) the entanglement with the meter kaon, necessary to perform marking or erasure, does not disturb the states $\left|K_{S}\right\rangle$ and $\left|K_{L}\right\rangle$ of the object kaon; (4) quantum erasure with entangled kaons allows one to restore the same interference phenomenon between the $K_{S}$ and $K_{L}$ propagation modes exhibited by a single kaon state produced as $K^{0}$ or $\bar{K}^{0}$.

An experimental test of the marking and erasure operations we have discussed is highly desirable and should be feasible at $\phi$-factories and $p \bar{p}$ machines. Actually, the CPLEAR collaboration [15] has already done part of the work required: the two experimental points (for $|\Delta \tau|=0$ and $1.2 \tau_{S}$ ) collected by this experiment reproduced the joint strangeness oscillations predicted by quantum mechanics [see Eq. (9)]. New measurements confirming with better precision these oscillations for a larger range of $\Delta \tau$ values, as well as the non-oscillating behaviour when "which width" information is in principle available [see Eq. (8)], are needed for a full complementarity test.

Work partly supported by EURIDICE HPRN-CT2002-00311, BFM-2002-02588, MA 7-1886/02 and INFN.

[1] M. O. Scully and K. Drühl, Phys. Rev. A 25, 2208 (1982).
[2] M. O. Scully, B.-G. Englert and H. Walther, Nature (London) 351, 111 (1991); ibid. Am. J. Phys. 67, 325 (1999).

[3] P. G. Kwiat, A. M. Steinberg and R. Y. Chiao, Phys. Rev. A 49, 61 (1994).

[4] B.-G. Englert, Phys. Rev. Lett. 77 (1996) 2154; B.-G. Englert and J. A. Bergou, Opt. Commun. 179, 337 (2000).

[5] S. Dürr, T. Nonn and G. Rempe, Phys. Rev. Lett. 81, 5705 (1998); ibid. Nature (London) 395, 33 (1998); S. Dürr and G. Rempe, Opt. Commun. 179, 323 (2000).

[6] Z. Y. Ou, L. J. Wang, X. Y. Zou and L. Mandel, Phys. Rev. A 41, 566 (1990).

[7] X. Y. Zou, L. J. Wang and L. Mandel, Phys. Rev. Lett. 67, 318 (1991).

[8] A. G. Zajonc et al., Nature (London) 353, 507 (1991).

[9] T. J. Herzog, P. G. Kwiat, H. Weinfurter and A. Zeilinger, Phys. Rev. Lett. 75, 3034 (1995).

[10] P. G. Kwiat A. M. Steinberg and R. Y. Chiao, Phys. Rev. A 45, 7729 (1992).

[11] T. Tsegaye and G. Björk, Phys. Rev. A 62, 032106 (2000).

[12] S. P. Walborn, M. O. Terra Cunha, S. Pádua and C. H. Monken, Phys. Rev. A 65, 033818 (2002).

[13] Y. Kim, R. Yu, S. P. Kulik, Y. Shih and M. O. Scully, Phys. Rev. Lett. 84, 1 (2000).

[14] A. Trifonov, G. Björk, J. Söderholm and T. Tsegaye, Eur. Phys. J. D 18, 251 (2002).

[15] A. Apostolakis et al., Phys. Lett. B 422, 339 (1998).

[16] "The Second Daథne Physics Handbook", edited by L. Maiani, G. Pancheri and N. Paver (INFN, Laboratori Nazionali di Frascati, 1995).

[17] A. Bramon and G. Garbarino, Phys. Rev. Lett. 88, 040403 (2002); ibid. 89, 160401 (2002).

[18] P. H. Eberhard, Nucl. Phys. B 398, 155 (1993).

[19] P. K. Kabir, "The CP Puzzle" (Academic Press, London, 1968).

[20] G. C. Ghirardi, R. Grassi and T. Weber, Proceedings of the Workshop on Physics and Detectors for Daథne, edited by G. Pancheri (INFN, Laboratori Nazionali di Frascati, 1991) p.261.

[21] R. A. Bertlmann and B. C. Hiesmayr, Phys. Rev. A 63, 062112 (2001); B. C. Hiesmayr, PhD thesis, University of Vienna, 2002.

[22] When $\Delta \tau=\tau_{l}-\tau_{r}=0$, each one of the two members of a surviving kaon pair has the same probability for being a $K_{S}$ or a $K_{L}$. For $\Delta \tau=\tau_{l}-\tau_{r}>0$ $(<0)$, the left (right) moving kaon is more probably a $K_{L}$ than its right (left) moving partner. With this additional information, a simple calculation shows that one has to identify as right going $K_{S}$ 's all kaons decaying between $\tau_{r}$ and $\tau_{r}+\Delta \tau_{r}$, where $\Delta \tau_{r}$ must satisfy $\Delta \Gamma \Delta \tau+\Gamma_{S} \Delta \tau_{r}+\ln \left(1-\mathrm{e}^{\Gamma_{\mathrm{L}} \Delta \tau_{\mathrm{r}}}\right)=0$; undecayed kaons have to be identified as $K_{L}$ 's. Then, the $K_{S}$ and $K_{L}$ misidentification probabilities coincide and reduce, as in the single kaon case, to a few parts in $10^{-3}$.

[23] Note that the presence of the signal polarization analyzer at $45^{\circ}$, which is essential to perform quantum erasure, does not alter by itself the interference-free situation obtained when the idler polarization analyzer is placed at $0^{\circ}$ or $90^{\circ}$.

[24] Z. Y. Ou, Phys. Lett. A 226, 323 (1997). 